\title{
Uso de benzodiazepínicos entre idosos: 0 alívio de "jogar água no fogo", não pensar e dormir
}

\section{Benzodiazepine use among elderly: the relief of "throwing water on the fire", not thinking and sleeping}

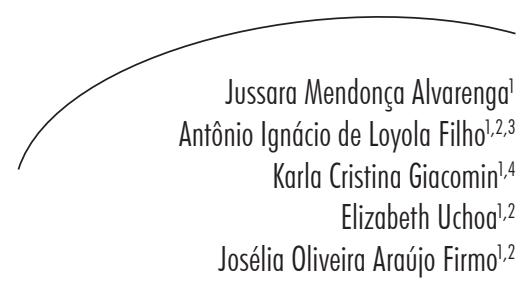

\section{Resumo}

Objetivo: Compreender a percepção e os significados que os idosos atribuem a suas experiências relacionadas ao uso prolongado de benzodiazepínicos. Métodos: Trabalho de abordagem qualitativa, de cunho antropológico, realizado junto a idosos participantes do Projeto Bambuí, estudo de base populacional sobre as condições de saúde da população idosa, realizado na cidade de Bambuí-MG. Foram realizadas entrevistas semiestruturadas com 22 idosos, sem comprometimento cognitivo e residentes na cidade de Bambuí-MG, que relataram uso de medicação benzodiazepínica. Resultados: No grupo pesquisado, o uso de benzodiazepínicos foi compatível com a definição de "padrão de uso crônico", variando de seis meses a 40 anos, sendo o medicamento mais utilizado o Clonazepam. A coleta e análise dos dados foram guiadas pelo modelo de signos, significados e ações. Emergiram como categorias: a utilização de um remédio "muito bom"; o remédio bom que "parece que vicia"; a (des)obediência à prescrição médica; e o alívio. Conclusão: Os idosos entrevistados justificam o uso crônico de benzodiazepínicos como um paliativo para lidar com dificuldades existenciais decorrentes de situações culturais, sociais e familiares, as quais precisam ser abordadas nos serviços de saúde.

\section{Abstract}

Objective: To understand the perception and meanings that elderly give to their experiences as related to prolonged use of benzodiazepines. Methods: Using an anthropological qualitative methodology of qualitative approach, the study was conducted among elderly participants in the Bambuí Project, a population-based study on the health

\footnotetext{
Fundação Oswaldo Cruz, Núcleo de Estudos em Saúde Pública e Envelhecimento. Belo Horizonte, MG, Brasil.

2 Fundação Oswaldo Cruz, Programa de Pós-graduação em Ciências da Saúde. Belo Horizonte, MG, Brasil.

3 Universidade Federal de Minas Gerais, Escola de Enfermagem, Departamento de Enfermagem Aplicada. Belo Horizonte, MG, Brasil.

4 Prefeitura de Belo Horizonte, Secretaria Municipal de Saúde, Coordenadoria de Atenção à Saúde do Idoso. Belo Horizonte, MG, Brasil.
}

Palavras-chave: Uso Crônico. Benzodiazepinas. Saúde do Idoso.

Envelhecimento. 
status of the elderly, in the city of Bambuí, Minas Geraisstate, Brazil. Semi-structured interviews were conducted with 22 elderly without cognitive impairment and residents in Bambuí-MG, who reported use of benzodiazepines. Results: In the study group, the use of benzodiazepines was compatible with the definition of "pattern of chronic use", ranging from 6 months to 40 years, and the most used drug was Clonazepam. The collection and analysis were guided by the model of signs, meanings and actions. These categories emerged: use of a "very good" drug; the good medicine that "seems addictive"; (dis)obedience to the prescription; and relief. Conclusion: The elderly respondents justify the chronic use of benzodiazepines as a palliative to deal with existential difficulties arising from cultural, social and family situations, which need to be addressed in the health services.
Key words: Chronic Use. Benzodiazepines. Health of the Elderly. Aging.

\section{INTRODUÇÃO}

Há mais de meio século, os benzodiazepínicos (BZD) figuram entre os medicamentos mais consumidos em todo o mundo. ${ }^{1}$ No entanto, estudos realizados a partir dos anos 1980 têm-lhes atribuído diversos efeitos indesejáveis, incluindo o risco de dependência, especialmente em caso de uso crônico. ${ }^{2}$ Os BZD de ação prolongada ou em altas doses, bem como seu uso crônico, foram considerados impróprios e associados a resultados adversos em idosos, ficando restritos a indicações clínicas específicas. ${ }^{3}$

Uma revisão sobre efetividade clínica, custobenefício e diretrizes sobre o uso de BZD em idosos concluiu que as evidências disponíveis sugerem maiores chances de eventos cognitivos e psicomotores adversos entre os usuários de BZD, ${ }^{4}$ tais como quedas e fraturas. Nenhum trabalho avaliou com precisão a segurança, eficácia clínica e custo-efetividade do uso de BZD no tratamento de ansiedade ou problemas de comportamento em idosos. Apesar disso, a prevalência do seu consumo nesse segmento etário mantém-se muito elevada - cerca de 30\% -, muitas vezes cronicamente, por muitos anos, ${ }^{5}$ sendo ainda maior entre idosos mais velhos. ${ }^{6,7}$

No Brasil, pessoas idosas são consumidoras frequentes de BZD, como evidenciam estudos epidemiológicos desenvolvidos em diferentes populações, sejam elas residentes em comunidade, com prevalência estimada de $22 \%,{ }^{8}$ ou usuárias de serviços de saúde, cuja prevalência chega a $30 \%$. Estudos qualitativos demonstram que usuários crônicos de BZD podem desenvolver dependência física e psicológica, conferindo a esses medicamentos atributos que suplantam aqueles decorrentes de sua ação farmacológica. ${ }^{10}$

Entretanto, esforços para interromper o uso de BZD permanecem abaixo do ideal, ${ }^{2}$ sendo necessária uma abordagem complementar para identificar outros fatores que possam contribuir para que uma retirada gradual possa ocorrer. ${ }^{11}$ Nesse sentido, é importante considerar que as práticas em saúde, inclusive o uso crônico de BZD, sofrem influência do contexto e da cultura em que a pessoa está inserida. ${ }^{12}$

Para Geertz, ${ }^{12}$ a cultura é um universo de símbolos e significados que permite a um grupo interpretar sua experiência e guiar suas ações. A experiência da doença e seu cuidado - inclusive o uso de medicamentos como os BZD - não podem ser considerados simples reflexo do processo patológico no sentido biomédico do termo, devendo ser concebidos como uma construção cultural que se expressa em "maneiras específicas de pensar e agir". ${ }^{3}$ Os profissionais, incluindo geriatras e gerontólogos, quase sempre se reportam ao saber biomédico, enquanto a percepção da população estaria ligada a uma rede de símbolos, articulando conceitos biomédicos e culturais.

Assim, o objetivo do presente estudo foi compreender a percepção e os significados que os 
idosos atribuem a suas experiências relacionadas ao uso crônico de benzodiazepínicos.

\section{METODOLOGIA}

\section{População estudada}

O estudo foi conduzido entre idosos participantes do Projeto Bambuí, estudo prospectivo de base populacional desenvolvido na sede desse município, situado a oeste de Minas Gerais. A coorte composta em 1997 totalizou 1.606 idosos ( $\geq 60$ anos), que correspondiam a $92 \%$ de toda a população nessa faixa etária. As entrevistas foram realizadas com idosos participantes do sétimo segmento da coorte.

O principal objetivo da coorte é identificar preditores da mortalidade, da hospitalização e da limitação física e cognitiva em idosos, o que possibilitará a identificação de condições e/ou de idosos-alvo para atividades de prevenção. Nos seguimentos anuais da coorte, acontecem entrevistas para coleta de informações; em alguns anos, há coleta de material biológico, eletrocardiograma e medidas antropométricas. Em todos os seguimentos, avaliaram-se o consumo de medicamentos (confirmado pela prescrição e/ou embalagem da medicação em uso) e a condição cognitiva da população idosa, por meio da aplicação do Mini-mental..$^{14}$ Mais detalhes podem ser pesquisados em Lima-Costa et al. ${ }^{15}$

À época do presente estudo, 1.113 idosos compunham a coorte, dos quais $25,2 \%$ estavam em uso de BZD. Foram critérios de inclusão: ser participante do Projeto Bambuí, relatar fazer uso de BZD e não apresentar comprometimento cognitivo à entrevista de seguimento anual. $\mathrm{O}$ uso crônico de BZD foi definido pela utilização de pelo menos uma dosagem do medicamento por cinco ou mais dias da semana, durante quatro meses ou mais. ${ }^{16}$ Para regular o tamanho da amostra, foi utilizado o critério de saturação, momento a partir do qual se identificam fatores operacionais de redundância e repetição de dados, bem como fatores teóricos de consistência e representatividade de elementos apresentados. ${ }^{17}$

\section{Coleta e análise de dados}

O modelo dos Signos, Significados e Ações, desenvolvido por Corin et al., ${ }^{18}$ foi utilizado na coleta e análise dos dados, buscando sistematizar os elementos do contexto que participam da construção da maneira de pensar e de agir dos idosos com relação ao uso de BZD.

O modelo tem sua origem na definição geertziana da cultura e busca identificar os diversos elementos do contexto que influenciam a concretização de lógicas culturais. ${ }^{12}$ Parte do princípio de que cada comunidade constrói de maneira específica seu universo de problemas, marcando alguns, privilegiando esta ou aquela explicação e encorajando certos tipos de reações. Esse modelo procura identificar os signos (comportamentos associados), os significados (explicações privilegiadas frente a esses signos), como também as reações que predominam no evento de saúde, o qual não é visto como um processo biomédico, mas percebido como uma construção cultural.

Para reconstruir o universo de representações (maneira de pensar) e de ações (maneiras de agir) associadas ao uso de BZD entre os idosos pesquisados, foram realizadas entrevistas domiciliares após apresentação e assinatura do Termo de Consentimento Livre e Esclarecido. As entrevistas foram gravadas e posteriormente transcritas. Foram iniciadas pela pergunta: " $O(a)$ senhor(a) fez uso de alguma medicação nos últimos três meses?" Em caso de resposta positiva, seguiu-se a pergunta: " $O(a)$ senhor(a) se lembra de quais foram essas medicacõoes? Pode me mostrar todas as receitas e/ou embalagens dos remédios que você está usando?". Com base nas respostas dadas às perguntas iniciais e as medicações identificadas, foram exploradas questões relacionadas ao uso e à percepção do idoso em relação ao BZD.

As entrevistas foram lidas atentamente, por repetidas vezes, com o objetivo de identificar unidades significativas e criar categorias analíticas, procedimento que propiciou a construção de um esquema de codificação. Em 
seguida, os textos foram marcados em função das categorias e subcategorias identificadas, sendo examinada a interação entre elas. Dessa forma, foram identificados os signos e os significados que os idosos atribuíam à utilização da medicação benzodiazepínica, bem como as ações relativas a esse uso.

\section{Aspectos éticos}

A pesquisa foi aprovada pelo Comitê de Ética em Pesquisa do Centro de Pesquisa René Rachou, da Fundação Oswaldo Cruz, segundo o protocolo no 18/2010 (CAAE: 0018.0.245.000-10).

\section{RESULTADOS E DISCUSSÃO}

Vinte e dois idosos foram entrevistados, sendo 18 mulheres e quatro homens. Todos os homens e a maioria das mulheres (10) estavam em uso de Clonazepam. Entre as mulheres também foram mencionados Bromazepam, Lorazepam e Diazepam. Todos eram usuários crônicos de BZD, sendo o tempo mínimo de consumo de seis meses e o máximo de quase 40 anos. Nenhum dos entrevistados teve a primeira prescrição e/ou posteriores em consulta com psiquiatra. A cidade também não dispunha de geriatras ou gerontólogos. Os prescritores foram clínicos, generalistas, cardiologistas ou neurologistas (neste caso, eles consultavam em outra cidade, de maior porte).

$\mathrm{Na}$ análise emergiram as seguintes categorias relativas à utilização do benzodiazepínico: a utilização crônica de um remédio "muito bom"; o remédio bom que "parece que vicia"; a (des) obediência à prescrição médica; e o alívio.

\section{A utilização crônica de um remédio "muito bom"}

Nesta categoria, os participantes fizeram uma avaliação positiva sobre os BZD, sintetizada no excerto: "É melhor ficar sem o arroz que ficar sem ele (BZD)". (F16)
Muitos idosos, embora fizessem uso crônico do remédio, desconheciam o nome do produto, identificando-o como "aquele de tarja preta", "o que precisa da receitinha azul", necessitando buscar a caixa de remédios ou da ajuda de terceiros. Alguns usaram mais de um tipo ao longo da vida; outros usavam o mesmo há décadas. Apenas uma mulher afirmou ter lido a bula e descoberto que a medicação seria inadequada, mas ainda assim continuou a utilizála. Uma entrevistada reconheceu: "Por que eu tomo? Eu não sei por que eu tomo. Porque o médico é que receitou". (F6)

Embora a introdução do medicamento se desse por indicação médica, nota-se o desconhecimento da sua indicação terapêutica, semelhante àquela demonstrada em estudo que avaliou o nível de conhecimento sobre a hipertensão arterial e a adesão ao tratamento entre idosos hipertensos de Bambuí-MG. Cerca de um quarto dos idosos desconhecia ser hipertenso; enquanto mais de um terço dos hipertensos não seguia o tratamento. ${ }^{19}$

No entanto, como o BZD somente é obtido mediante prescrição médica, uma mulher explicou como conseguia a receita:

Quando preciso de uma receita, eu nem vou lá (ao serviço de saúde). Ela [funcionária do serviço] já sabe. Eu telefono, ele entrega para a secretária dele, uma senhora que mora bem aqui pertinho, ela tra乏pra mim e eu continuo. (F1).

Outra mulher reclamou: "Se tivesse outro farmacêutico (que vendesse o BZD) sem consulta médica eu ia comprar dele. Eles não vendem" (F7).

$\mathrm{Na}$ maioria dos relatos, sequer houve necessidade de realizar consultas médicas regulares, pois "qualquer um" fornecia a receita, estando o acesso à prescrição dependente mais das relações pessoais e familiares e/ou da mediação de funcionárias do próprio serviço de saúde. ${ }^{20}$ Por muito tempo, o uso de psicofármacos pela população não foi adequadamente controlado pelas autoridades sanitárias brasileiras, daí 
a incompreensão da exigência de prescrição médica. Resultado semelhante foi observado junto a trabalhadores rurais brasileiros. ${ }^{21}$

Tampouco foram observadas falas sugestivas de orientação médica no sentido de utilizar BZD por um prazo definido ou de aconselhamento para reduzir e/ou interromper seu uso. Essa questão reitera os resultados de Iliffe et al., ${ }^{22}$ que investigaram idosos londrinos e verificaram que nenhum profissional os alertou quanto aos riscos e danos do uso continuado de BZD. Para Halme et al., ${ }^{2}$ resultados de intervenções direcionadas para farmacêuticos e médicos visando interromper prescrições de BZD são muito limitados. Explicariam tal fracasso: a percepção da questão como não sendo uma prioridade clínica, pelos profissionais, e a minimização de seus efeitos adversos, pelos consumidores, os quais transferem a responsabilidade dessa prescrição exclusivamente para seus médicos. ${ }^{2}$ Esta entrevistada justifica:

Ah, eu sou muito nervosa. Se eu ficar nervosa aí
acabou eu não durmo. Tem dia que a gente fica nervosa
à toa, né? Outra hora a gente pensa muito assim na
velhice da gente, nos filhos esparramados, né? Uns
tem uns problemas, outros, (têm) outros. A gente se
preocupa. (F13)

Para Anthierens et al., ${ }^{23}$ talvez por considerarem que a situação está fora de controle, os pacientes usam duas estratégias para justificar o uso de BZD: maximizar seus problemas e minimizar seu uso prolongado.

Entre os homens entrevistados, os signos relativos ao motivo de continuar utilizando essa medicação estavam relacionados a problemas para dormir ou labirintite. Entre as mulheres, foram identificados: dificuldades relativas ao sono, nervosismo, solidão, sintomas depressivos ("não comia", "só pensava coisa ruim", "ruindade", “esgotei”), preocupação com problemas familiares (alcoolismo de filhos, cuidado do marido com câncer), existenciais, ou reação ao luto (perda de marido, de filhos e de familiares).
Tal resultado confirma que fatores interpessoais, sociais e relacionados ao desenvolvimento do sujeito podem contribuir para a cronicidade do uso e, eventualmente, de dependência. ${ }^{20}$

No universo pesquisado, muitos entrevistados relataram opiniões de amigos, vizinhos e familiares sobre o uso dessa medicação, como se observa nestas falas: "Porque muita gente falava: esse remédio é muito bom para dormir”. (F4).

Eles falam que às vezes ele (o BZD) pode até estar me fazendo mal [...] mas não é dele não, gente. Tem muitos anos que eu tomo ele. Eu tenho vizinho que fala: 'às vezes é mal desses remédios que você toma”". (F16).

Assim, conforme evidenciado na literatura, "o uso de benzodiazepínicos não se restringe a uma relação biológica de seus efeitos, mas a uma interação com o cultural e o social", ${ }^{11}$ não ficando restrito a uma relação entre médico e paciente. ${ }^{24}$

Em Bambuí-MG, no entanto, as opiniões favoráveis aos BZD foram acolhidas, mas as contrárias mostraram-se insuficientes para interromper seu uso. Perguntada se deixaria de tomar a medicação, esta entrevistada afirmou: "Ah, não, eu não vou deixar, não (de tomar). Acho que vou usar eternamente. Até morrer" (F16).

Se o início de uso se dá pela prescrição médica, continuar ou não utilizando deriva do julgamento do medicamento por parte de quem o utiliza, com base na experiência, positiva ou negativa, que obteve com ele. No presente trabalho, apenas uma mulher afirmou ter interrompido o uso da medicação mediante a recusa das filhas em adquiri-la, após considerarem a orientação do farmacêutico:

\footnotetext{
As meninas ainda falou assim: "Ih, mãe, aquele remédio da faixa preta?"' Eu falei: "isso aí eu não sei não. Ele é?" [relata a opinião do homem da farmácia ao contraindicar o uso do benzodiazepinico]. Ele até falou para a menina: "sua mãe é de idade. É idosa". "Ela [a filha] só falou que não ia comprar mais, dai não comprou mais não. (F7).
} 
Ainda assim, a entrevistada deixou transparecer que a decisão de interromper o uso da medicação não foi por recomendação médica, e sim das filhas. Quanto à razão para sua continuidade, predominava o temor de que os sintomas retornassem, se intensificassem, ou comprometessem o desempenho das atividades cotidianas. Essa senhora explicou sua necessidade de continuar a usar a medicação:

[O filho alcoolista] parou de beber e eu melhorei. [...] Então en queria experimentar assim: se eu passar um dia sem tomar para ver se eu durmo. Mas eu tenho [medo] só de pensar que eu não vou tomar, eu acho que eu não vou dormir [...] como é que eu vou fazer? No outro dia en tenho que trabalhar, fazer comida, arrumar a casa. (F12).

De modo semelhante, significativa dependência psicológica, concomitante à subestimação ou negação de potenciais efeitos colaterais dos BZD, também foi observada em estudos realizados junto a idosos norteamericanos, com grande resistência por parte deles, especialmente os mais velhos, à suspensão da medicação. ${ }^{10,25}$

\section{0 remédio bom que "parece que vicia"}

Esta categoria apresenta a avaliação que fazem sobre o uso crônico do remédio. Duas entrevistadas reconheceram o uso prolongado, mas ponderaram:

[Por que a senhora continua usando?] Eu acho que esses remédios é tipo de uma droga, porque a pessoa que fuma porque o cigarro é uma droga, eu nunca coloquei na boca. O remédio eu acho que é a mesma coisa. Você 'vicia' naquele remédio. (F9)

A pessoa acostuma e vicia. Talvez se tirasse poderia também nem fazer mal, fazer bem. (F1)

Apesar de considerarem o "vício", não atribuem gravidade a esta condição, nem acreditam que ela possa necessariamente ocorrer entre eles, como ilustrado na fala deste homem:
Porque muita gente fala que está em dependência deste remédio. Eu não estou. Eu não tomo ele e durmo a mesma coisa, não tem problema nenbum. (M2).

No campo do conhecimento biomédico, define-se a dependência de BZD em idosos como um processo contínuo que pode causar problemas de gravidade variável no indivíduo, e que depende das circunstâncias pessoais, interpessoais e sociais. ${ }^{26}$ Em adultos, a dependência de fármacos é definida pela presença de um agrupamento de sintomas cognitivos, comportamentais e fisiológicos, decorrentes do uso continuado de uma substância, que geralmente resulta em tolerância, abstinência e comportamento compulsivo (DSM-IV-TR). ${ }^{27}$

Entre idosos ela seria pouco identificada, uma vez que muitos tendem a não relatar seu uso e até 15\% ocultam ou negam o uso de psicotrópicos. $^{28}$ Nas lógicas conceituais subjacentes ao comportamento concreto dos entrevistados associado ao uso prolongado do BZD, configura-se uma referência amenizada ao termo "dependência", enquanto a palavra "vício" é utilizada apenas com o sentido daquilo que é repetitivo.

Ademais, persistem dúvidas quanto aos fundamentos da natureza dessa dependência, ${ }^{26}$ pois muitos dos critérios utilizados para caracterizá-la não se aplicam ao grupo etário idoso, em razão de mudanças decorrentes do padrão de uso de medicamentos na velhice.

\section{(Des)obediência à prescrição médica}

Quanto à obediência à prescrição médica, quando do uso crônico de BZD, os idosos respeitaram a dose prescrita; para reduzir, negociaram com o médico ou fizeram-no por conta própria. Apenas quando relacionam o uso do BZD a um efeito indesejável (exagerado) ou perigoso, a prescrição pode ser desobedecida. Tomando por base o efeito e a potência da medicação - referindo-se ao número de 
miligramas do fármaco -, uma mulher explicou por que, por conta própria e sem comunicar ao profissional, decidiu reduzir a dose prescrita:

Era para mim tomar meio comprimido. Ai eu tomei, tomei meio, [clonazepam] mas eu vi que não me fez. bem. Eu dormi demais. [...] este aqui [bromazepam] en vou te mostrar o que en fir: Se duas miligramas (de clonazepam) estava muito forte, ele receitou ${ }_{0}$ bromazepam] de três, mandou tomar um, olha o que en fir. [...]. Ele mandou tomar um deste [bromazepam] e um deste [amitriptilina]. Se eu tivesse tomado estaria lá na cidade dos pés juntos. Que é o cemitério. IA senhora voltou depois no médico ou não?] Não. Abandonei por minha conta. [E voltou para o clonazepam?] É. Por minha conta eu dividi ele em quatro partes. Então são um quarto de 2 mg. (F12)

Exceto para um entrevistado que referiu aumento pontual da dosagem do Clonazepam ao se sentir muito nervoso, todas as alterações posológicas promovidas pelos idosos foram de redução da dosagem. Nenhum uso abusivo do medicamento foi identificado. Padrão semelhante foi observado em estudo que investigou o consumo de BZD na população idosa residente em Diamantina-MG, em que nenhuma das posologias relatadas pelos entrevistados se enquadrou nos critérios que definem a superdosagem. ${ }^{26}$

Uma mulher admitiu sua vontade de parar de tomar o BZD:

Eu tenho vontade [de reduzir a dose], já falei com ele: "doutor, eu não aguento essa quantidade"... Io médico diz]. "Não estou te perguntando se tem ou não tem, vai comprar o remédio e vai tomar". (F10).

Esta mulher descreve como conseguiu:

Eu também não sou de ouvir qualquer pessoa não. [... ou eu vou pela minha intuição, ou então eu vou no médico. Principalmente em sala de espera, você ouve muito. Um receita pra um, receita pra outro. [...] Eu usei [clonazepam] durante uns cinco anos seguidos, um médico que tratou me passou. Depois eu achei que estava ficando muito desanimada, mal estar demais, aí eu interrompi. Mas, assim: fui no médico. (F8).
Considerando que em cada ação médica "há um conduzir o doente, no qual o diálogo e a comunhão desenvolvida pelo diálogo entre o médico e o paciente desempenham papel decisivo", ${ }^{29}$ em Bambuí-MG, nem sempre o profissional se dispôs a ouvir quem questionou sua prescrição. A obediência à prescrição médica revela-se mais forte do que a percepção de que o medicamento não seja mais necessário, como no extrato que se segue:

[...] eu posso estar louca de sono, mas eu vou lá, pego os remédios direitinho e tomo direitinho. [...] tomo aquela quantidade certinha que o médico mandou tomar. (F11).

Atitude semelhante diante da autoridade médica emergiu em outro estudo sobre a percepção da saúde e incapacidade na velhice, em que se observa que os idosos agem como se o médico conhecesse melhor suas necessidades de saúde do que eles próprios, permanecendo a prática médica inquestionável. ${ }^{30}$

0 alívio

Para os entrevistados, o BZD representa um suporte para lidar com as dificuldades da vida e com os medos na velhice:

Porque para gente velho, um tranquilizante, a gente suporta melhor as dores, os problemas [...]. Não é que eu seja nervosa, é que eu tenha necessidade. (F8).

É, tem dias que a gente tá contrariada. Ai eu tomo um (comprimido de clonazepam) é a mesma coisa que jogar água no fogo. [...] porque família dá problema. (F19).

Vou comprar ele [o remédio] que eu tomo, não penso e durmo. (F4).

Por trás do "não pensar" nos problemas existenciais que os incomodam, está o desejo de dormir uma noite de sono que lhes amortecerá os sentidos e tornará a realidade menos angustiante ou mais suportável. Dormir é uma necessidade fisiológica: para a pessoa que dorme, a noite dura apenas um momento. Meadows ${ }^{31}$ insiste no fato 
de que o sono não é uma "inação social", mas um tempo de interação, fruto de uma negociação entre seus próprios desejos, expectativas e papéis sociais e aqueles dos outros.

Pode-se perguntar - como fazem os médicos - se é a dimensão fisiológica da noite que provoca uma alteração do estado físico ou se é sua dimensão simbólica, pois a noite altera nosso estado de consciência e de vigília. ${ }^{32}$ A noite também possui uma dimensão cultural fortemente demarcada, ${ }^{33}$ ao contrapor notadamente o comportamento de pessoas vivendo a juventude e a velhice.

Na juventude, a noite é vivida no espaço de fora, em grupo, assumindo comportamentos arriscados - momento especial da transgressão, que serve para o alívio do estresse, a compensação e, portanto, um papel de reparação, de coesão social e para possibilitar ao jovem encontrar um equilíbrio entre racionalidade e irracionalidade. $\mathrm{Na}$ velhice, a noite é um tempo vivido não em grupo, nem no exterior, mas na solidão da própria casa ou em instituição.

Assim, usualmente, a noite está proibida aos mais velhos como tempo de vida, pois não visa à transgressão, mas prenuncia a morte. Nessa hora, a noite leva-os a lidar com questões dolorosas, acompanhada por vezes de pensamentos tristes ou de preocupações que aparecem quando o velho não consegue achar o sono e frequentemente vêse relegado à solidão, à angústia e ao sofrimento. ${ }^{33}$

Uma idosa afirmou:

Esta noite depois das três horas que eu tive insônia, não dormi um segundo. Não sei por quê. Não sei se é porque eu fico pensando assim a vida, a gente fica sozinha, tem dia que não aparece uma pessoa aqui para conversar comigo! Quando aparece, tem vez que é pra amolar. (F11).

Portanto, bem mais interessante para o estudo antropológico, é a noite que nunca termina, não a noite escura de sono, mas a noite em claro da insônia, ${ }^{31}$ pois, como lembra Gadamer, ${ }^{29}$ "uma das grandes forças curativas da vida é o fato de, todas as noites, entregarmo-nos ao sono curativo do esquecer. Não poder esquecer é um sofrimento pesado". Como fazer para esquecer ou não pensar nas dificuldades cotidianas da vida, para as quais não há qualquer previsão de solução próxima ou futura?

Um homem esclareceu: "Ainda dá esse desatino, mas no espaço de meia hora, ele já faz efeito". (M4)

Ao discutir o caráter oculto da saúde, Gadamer $^{29}$ afirma que "toma-se algo para combater a doença e, então ela desaparece". Porém, no que tange à saúde mental, esse autor acredita que os psicofármacos possam introduzir uma forma de agressão totalmente nova, ao retirar da pessoa suas próprias indisposição e perturbação mais profundas.

$\mathrm{Na}$ cultura local, percebe-se que o BZD representa uma alternativa para lidar com os problemas da vida, legitimamente avalizada pelo saber biomédico, com a garantia do alívio rápido - "em meia hora" -, de apagar o incêndio - “jogar água no fogo" - e possibilitar no sono, o esquecimento. Há que se considerar que as palavras da noite têm um peso diferente daquelas do dia. ${ }^{33}$ Afinal, não se estará acordado para lidar com as questões da velhice, dos filhos; do medo da morte, do estresse cotidiano, da solidão, das dores e problemas. Talvez essa não seja a melhor nem a mais adequada ou a menos arriscada maneira de lidar com essas questões, mas aquela possível.

Por se tratar de pesquisa qualitativa, os resultados não podem ser generalizados, ${ }^{34}$ mas considera-se que os resultados aqui apresentados permitem uma relevante compreensão acerca dos motivos que sustentam a utilização crônica de BZD na visão dos idosos.

\section{CONSIDERAÇÕES FINAIS}

No universo pesquisado, os idosos atribuem significados e interpretações culturalmente associados a suas experiências quanto ao uso 
prolongado de benzodiazepínico, emergindo lógicas distintas daquelas que descrevem a prescrição médica do fármaco, revelando a maneira como percebem e interpretam essa experiência no seu cotidiano.

A falta de sono, que justifica o uso de benzodiazepínico, revela situações culturais, sociais e familiares que precisam ser abordadas de forma coordenada nos serviços de saúde. Cabe refletir se a prescrição do benzodiazepínico para fazer dormir e acalmar pessoas idosas angustiadas, aflitas, solitárias, não estaria reduzindo a oportunidade de escuta dos problemas existenciais por que passam essas pessoas, ou se representa em si uma comodidade para o profissional diante das limitações do cuidado e do serviço.

\section{REFERÊNCIAS}

1. Bueno JR. Emprego clínico, uso indevido e abuso de benzodiazepínicos-uma revisão. Rev Debates Psiquiatr 2012;2(3):6-11.

2. Halme AS, Beland SG, Preville M, Tannenbaum C. Uncovering the source of new benzodiazepine prescriptions in community-dwelling older adults'. Int J Geriatr Psychiatr 2013;28(3):248-55.

3. American Geriatrics Society. The American Geriatrics Society Updated Beers Criteria for potentially Inappropriate Medication use in Older Adults. J Am Geriatr Soc 2012;60(4):616-31.

4. McIntosh B, Clark M, Spry C. Benzodiazepines in older adults: a review of clinical effectiveness, cost-effectiveness, and guidelines [Internet]. Ottawa: Canadian Agency for Drugs and Technologes in Health; 2011[acesso em jan 2012]. Disponível em: http://www.ncbi.nlm.nih.gov/books/NBK174561/ pdf/TOC.pdf .

5. Voyer P, Préville M, Cohen D, Berbiche D,Bélard SG. The prevalence of benzodiazepine dependence among community-dwelling older adult users in Quebec According to typical and atypical criteria. Can J Aging 2010;29(2):205-13.

6. Egan M, Moride Y, Wolfson C, Monette J. Long-term continuous use of benzodiazepine by older adults in Quebec: prevalence, incidence and risk factors. J Am Geriatr Soc 2000;48(7):811-6.
O presente trabalho enfatiza a necessidade de abordar o uso prolongado e indiscriminado de benzodiazepínico por idosos no serviço de saúde, junto aos profissionais e à comunidade. Certamente, esta não é uma tarefa restrita apenas a uma profissão ou a um contexto social, familiar, profissional. Ela demanda o cuidado do profissional médico, bem como intervenções psicológicas e sociais, incluindo buscar a valorização do sujeito idoso, promover a compaixão diante da impotência que o ser humano experimenta ao longo da vida, mas também proporcionar outras formas de alívio, de convívio, de esperança. Isso passa pela criação de perspectivas para as condições de vida e de cuidado (dos idosos) e das condições de trabalho (dos profissionais) que favoreçam a escuta e aliviem o sofrimento de todos.

7. Préville M, Bossé C, Vasiliadis HM, Voyer P, Laurier C, Berbiche D, et al. Correlates of potentially inappropriate prescriptions of benzodiazepines among older adults: results from the ESA study. Can J aging 2012; 31(3):313-22.

8. Alvarenga JM, de Loyola AI Filho, Firmo JOA, Lima-Costa MF, Uchôa E. Prevalence and sociodemographic characteristics associated with Benzodiazepines use among community-dwelling older adults: results from the Bambuí study. Rev Bras Psiquiatr 2007;30(1):7-11.

9. Nordon DG, Akamine K, Novo NF, Hubner CvK. Características do uso de benzodiazepínicos por mulheres que buscavam tratamento na atenção primária. Rev Psiquiatr Rio Gd Sul 2009;31(3):152-8.

10. Cook JM, Biyanova T, Masci C, Coyne JC. Older patient perspectives on long-term anxiolytic benzodiazepine use and discontinuation: a qualitative study. J Gen Intern Med 2007;22(8):1094-1100.

11. Mendonça RT, Carvalho ACD. O consumo de benzodiazepínicos por mulheres idosas. SMAD, Rev Eletrônica Saúde Mental Álcool Drog 2005;1(2):1-13.

12. Geertz C. The interpretation of cultures. NewYork: Basic Books; 1973.

13. Uchôa E, Vidal JM. Antropologia médica: elementos conceituais e metodológicos para uma abordagem da saúde e da doença. Cad Saúde Pública 1994;10(4):497-504 . 
14. Folstein MF, Folstein SE, McHugh PR. "Mini-Mental State": a practical method for grading the cognitive state of patients for the clinician. J Psychiatr Res 1975;12:189-98.

15. Lima-Costa MF, Uchôa E, Guerra HL, Firmo JOA, Vidigal PG, Barreto SM. The Bambuí Health and Ageing Study (BHAS): methodological approach and preliminary results of a population-based cohort study of the Brazil. Rev Saúde Pública 2000;34(2):126-35.

16. Llorente MA, David D, Golden AG, Silverman MA. Defining patterns of benzodiazepine use in older adults. J Geriatr Psychiatr Neurol 2000;13(3):150-60.

17. Fontanella BJB, Ricas J, Turato ER. Amostragem por saturação em pesquisas qualitativas em saúde: contribuições teóricas. Cad Saúde Pública 2008;24(1):17-27.

18. Corin E, Uchôa E, Bibeau G, Koumare B. Articulation et variations des systèmes de signes, de sens et d'actions. Psychopathol Afr 1992;24:183-204.

19. Firmo JOA, Lima-Costa MF, Uchôa E. Projeto Bambuí: maneiras de pensar e agir de idosos hipertensos. Cad Saúde Pública 2004;20(4):1029-40.

20. Alvarenga JM, Giacomin KC, Loyola AI Filho, Uchôa E, Firmo JOA. Uso crônico de benzodiazepínicos entre idosos: "nervoso controlado", alívio garantido. Rev Saúde Pública. No Prelo.

21. Gage SB, Bégaud B, Bazin F, Verdoux H, Dartigues JF, Péres A, et al. Benzodiazepine use and risk of dementia: prospective population based study.BMJ 2012;345:1-12.

22. Iliffe S, Curran HV, Collins R, Yuen Kee SC, Fletcher S, Woods B. Attitudes to long-term use of benzodiazepine hypnotics by older people in general practice: findings from interviews with service users and providers. Aging Ment Health 2004;8(3):242-8.

23. Anthierens S, Pasteels I, Habraken H, Steinberg P, Declercq T, Christiaens T. Barriers to nonpharmacologic treatments for stress, anxiety, and insomnia Family physicians' attitudes toward benzodiazepine prescribing. Can Fam Physician 2010;56(11):398-406.
24. Auchewski L, Andreatini R, Galduróz JCF, Lacerda RB. Avaliação da orientação médica sobre os efeitos colaterais de benzodiazepínicos. Rev Bras Psiquiatr 2004;26(1):24-31.

25. Landi F, Onder G, Cesari M, Barillaro C, Russo A, Bernabei RS, et al. Psychotropic medications and risk falls among community - dwelling frail older people: an observational study. J Gerontol Ser A Biol Sci Med Sci 2005;60(5):622-626.

26. Telles PC Filho, Lima AMJ, Chagas AR, Durão MAS, Pinheiro MLP. Utilização de benzodiazepínicos por idosos de uma estratégia de saúde da família: implicações para a enfermagem. Esc Anna Nery Rev Enferm 2011;15(3):581-6.

27. American Psychiatric Association. DSM 4: Manual diagnóstico e estatístico de transtornos mentais. 4. ed. Porto Alegre: Artmed; 2002.

28. Martin P,Tamblyn R, Ahmed S, Tannenbaum C. A drug education tool developed for older adults changes knowledge, beliefs and risk perceptions about inappropriate benzodiazepine prescriptions in the elderly . Patient Educ Couns 2013;92(1):81-7.

29. Gadamer HG. O caráter oculto da saúde. Petrópolis: Vozes; 2006.

30. Moraes GVO. Influência do saber biomédico na percepção da relação saúde/doença /incapacidade em idosos na comunidade [dissertação]. Belo Horizonte: FIOCRUZ, Centro de Pesquisas René Rachou; 2012.

31. Meadows R. The negotiated night: an embodied conceptual framework for the sociological study of sleep. Sociol Rev 2005;53(2):240-54.

32. Puijalon B. Edito. Gérontol soc 2006;1(116):10-12.

33. Henchoz K, Lalive d'Épinay C. Le sommeil et les significations de la nuit dans la grande vieillesse. Gérontol soc 2006;1(116):25-44.

34. Minayo MCS, Sanches O. Quantitativo-qualitativo: oposição ou complementaridade? Cad Saúde Pública 1993;9(3):239-62. 